\title{
Idiopathic Trapeziometacarpal Joint Osteoarthritis: Cause, Prevention and Treatment
}

\author{
Hormoz Zahiri ${ }^{1,2}$; Christopher Zahiri ${ }^{2}$; Mansour Eghbali ${ }^{2,}$ \\ ${ }^{1}$ University of Southern California (USC), Los Angeles, California, USA \\ 2 International Orthopedic Center for Joint Disorders, Beverly Hills, California, USA \\ ${ }^{*}$ Corresponding author: Mansour Eghbali, International Orthopedic Center for Joint Disorders, Beverly Hills, California, USA. Tel: +98-9131413175, Fax: +98-2133542020, \\ E-mail: mansoureghbali@gmail.com \\ Received: June 6, 2015; Revised: June 20, 2015; Accepted: July 18, 2015
}

\begin{abstract}
Background: Idiopathic osteoarthritis of the trapeziometacarpal(TMC) joint is common. Spontaneous attenuation of either the anterior oblique ligament (Beak ligament) or the dorsoradial ligament has been reported as the cause of the joint laxity, resulting in subsequent wear and tear of the articular cartilages. So far, no cause has been reported for the spontaneous attenuation of these key supporting ligaments of the TMC joint and no effective method of prevention or treatment has been reported for the early stages of the condition. Objectives: The authors reported a conservative method for the prevention as well as reversal of the early stages of the joint instability before the process of wear and tear sets in and the condition progresses towards osteoarthritis.

Materials and Methods: A retrospective study was performed on 17 patients with early stages of idiopathic TMC joint osteoarthritis. The patients were treated through a conservative management protocol with an average follow-up of 28.4 months. The protocol consisted of performing specifically devised isometric exercises and a functional abduction brace.

Results: The result of the conservative treatment was postural correction of the thumb, anatomical stability of the TMC joint and resolution of the symptoms as well as findings including pain, swelling and joint dorsoradial translation. The mean pain score of the patients was 3.6 before the treatment was begun and was zero after the treatment was completed.

Conclusions: The presented conservative method is recommended for either prevention or reversal of the early stages of TMC joint instability before the osteoarthritic changes set in.
\end{abstract}

Keywords: Trapeziometacarpal Joint; Osteoarthritis; Conservative Treatments; Prevention

\section{Background}

Idiopathic osteoarthritis of the Trapeziometacarpal (TMC) joint is common. Spontaneous attenuation of either anterior oblique ligament (Beak ligament), as described by Lane and Eaton et al. (1) or dorsoradial ligament, as described by Lin et al. (2) have been considered to be the initial causes of the joint instability, which result in the subsequent wear and tear of the articular cartilages (2-6).

No effective method of prevention or treatment of the early stages of the condition has been so far presented (7-10).

This condition appears in 16\% of middle-aged females and $5 \%$ of middle-aged males (4). It also appears in those who perform job activities that require repetitive side pinch of the thumb.

Biomechanically, TMC join is a double ginglymus joint that allows the abduction/adduction, flexion/extension and apposition (pronation) of the thumb (11-13). The stability of the joint during the full range of motion is provided by a "force nucleus", formed by four ligaments (ulnar collateral ligament, first intermetacarpal ligament, anterior oblique ligament and dorsoradial ligament), the most important of which is the dorsoradial ligament. During the range of motion, these ligaments become taught in a sequential way to maintain the joint stability at all times. The dynamic stability of the TMC joint is not guaranteed by the joint configuration or the static resistance of the ligaments. It is the dynamic force of the muscles crossing the joint that is essential for a strong and durable stability $(14,15)$.

The abductor pollicis longus and extensor pollicis brevis (extrinsic muscles) and opponens pollicis and abductor pollicis brevis (intrinsic muscles) are the joint dynamic stabilizers (16-19). Their stabilizing force maintains the TMC joint toward extension, abduction and apposition, which protects the joint from the dorsoradial translation force. However, there is a joint destabilizing force provided by the adductor pollicis (an intrinsic muscle) which levers the base of the first metacarpal in the direction of its dorsoradial translation. This is the same force that radially displaces the shaft of the first metacarpal in a Bennet fracture. The destabilizing force of the adductor pollicis is efficiently neutralized by the strength of the stabilizing muscles.

Copyright (C) 2015, Iran University of Medical Sciences. This is an open-access article distributed under the terms of the Creative Commons Attribution-NonCommercial 4.0 International License (http://creativecommons.org/licenses/by-nc/4.0/) which permits copy and redistribute the material just in noncommercial usages, provided the original work is properly cited. 
Pathomechanically, attenuation of the key ligament initiates the joint laxity and the gradual dorsoradial translation of the proximal end of the first metacarpal over the trapezium. As a result, laxity of the joint capsule appears. Clinically, during power grasping, a normal thumb is flexed over clenched fingers in apposition, similar to a fist making (Figure $1 \mathrm{~A}$ ). However, power grasping in patients and individuals predisposed to TMC joint lax- ity is performed with the thumb resting on the side of the clenched fingers, similar to a side-pinch (Figures 2 $A$ and $2 B$ ) with their hand dropped into an ulnar deviated position when at rest (Figure $3 \mathrm{~A}$ ). The patients' TMC joint, in time, progresses toward a subluxated position with the base of the first metacarpal protruding as a bony prominence at the dorsoradial aspect of the joint (Figure 3 B).

Figure 1. Normal Thumb Position in Power Grasping
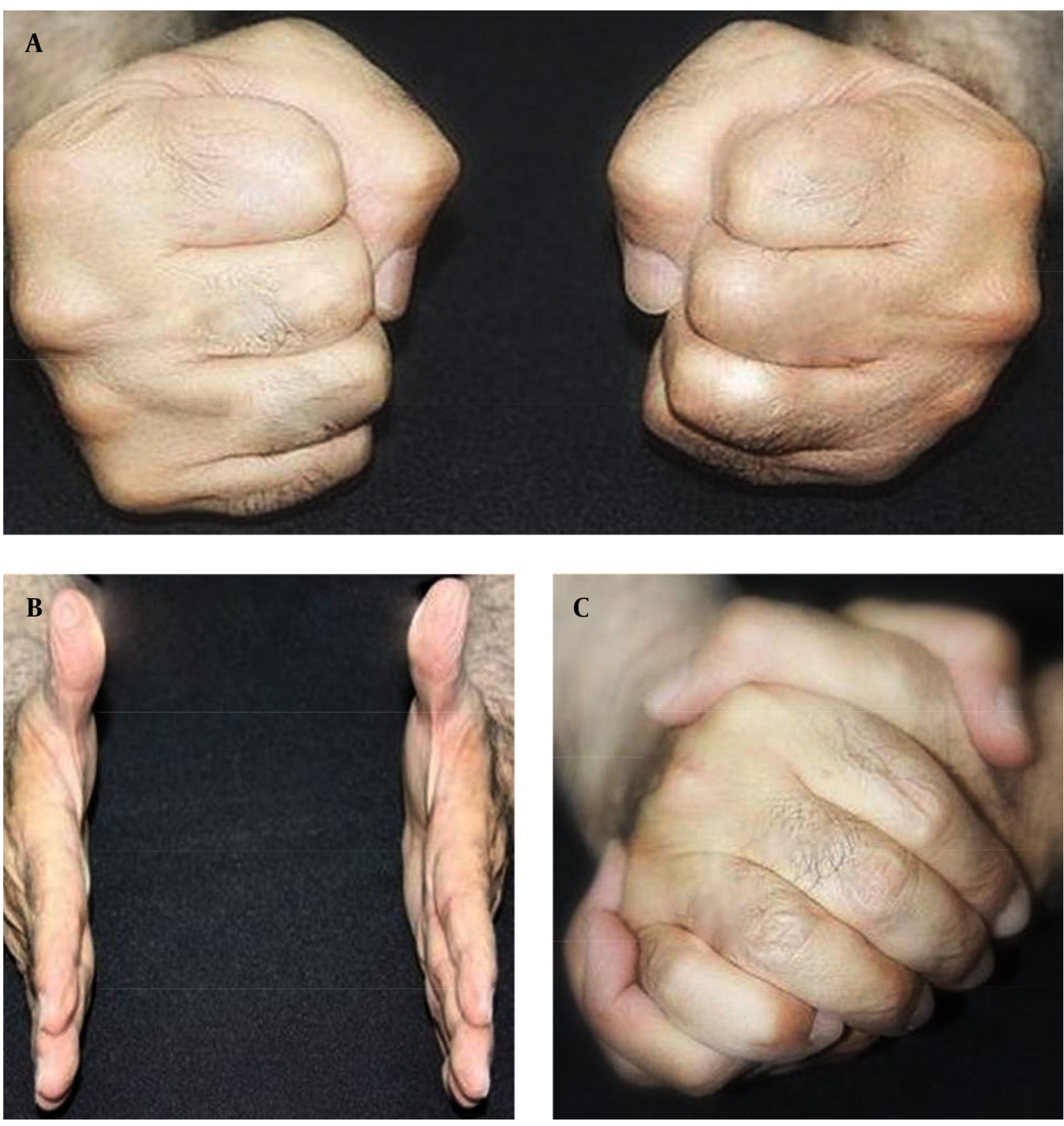

A, correct fist making with thumbs in full apposition/abduction/flexion; this also demonstrates the "fist making" exercise; B, sustained forceful thumb abduction exercise to follow each episode of "fist making"; C, "self-shake hands" exercise for the left thumb. As noted, the four fingers of the right hand provide the maximum abduction of the left thumb through separation of the thumb from the index finger. 
Zahiri Het al.

This characteristic fist making with adducted position of the thumb performed by potential individuals for TMC joint laxity (Figures $2 \mathrm{~A}$ and $2 \mathrm{~B}$ ) can be used as a clinical diagnostic test to predict a pending condition. During the examination, the patients are asked to make a fist with their bilateral hands. The normal fist making can be seen in Figure $4 \mathrm{~A}$. The abnormal habitual fist making can be seen in Figure $2 \mathrm{~A}$.

To prevent or treat the early stages of the TMC joint instability and subluxation, a two-pronged approach was adopted. One approach was to avoid stretching of the key ligament for which a low profile functional brace was designed and used (Figure 4). The brace would allow the normal function of the involved thumb and hand while maintaining the TMC joint in reduction at all times. The patients were advised to use the brace at day time as well as at night time. The other approach was to strengthen dynamic stabilizers of the TMC joint for which specific isometric exercises were devised and used by the patients, as follows:

Figure 2. Views of Thumb in Adducted Position in Individuals Predisposed to Trapeziometacarpal Joint Laxity
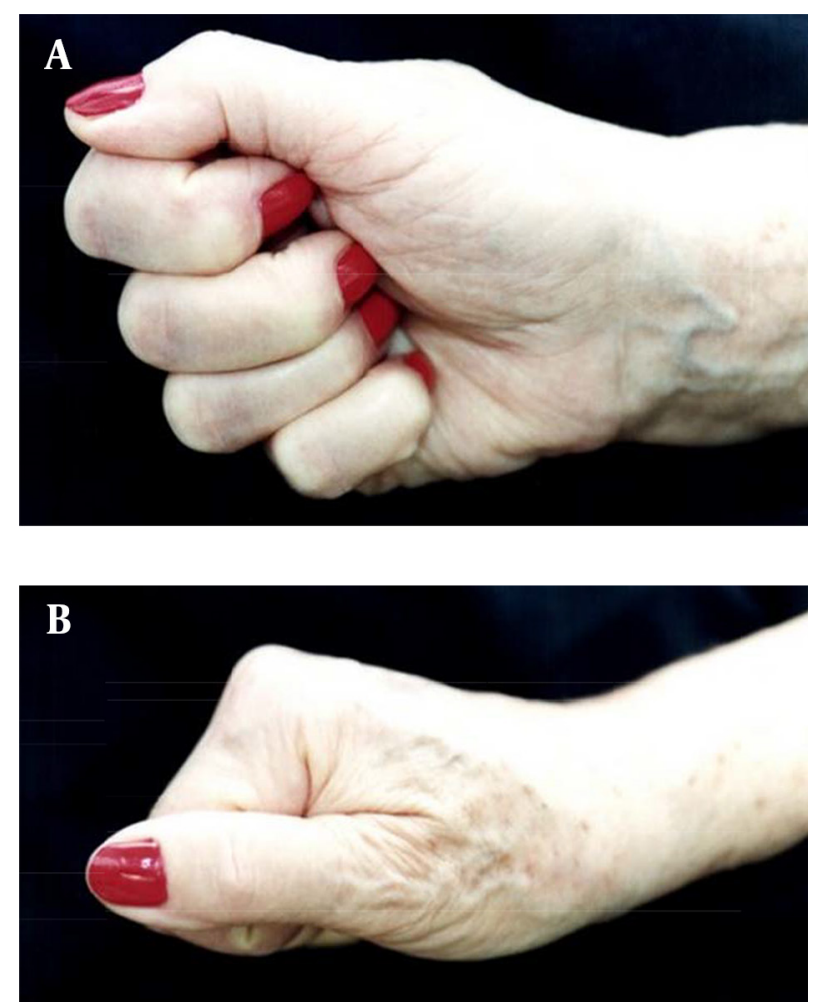

A, anterior view of a habitual fist making with the thumb resting in adducted position, similar to a side-pinch; B, lateral view of the adducted position of the thumb.
1. Fist making exercise (Figure $1 \mathrm{~A}$ ); 2 . Self-hand shake exercise (Figure $1 \mathrm{~B}$ ); 3. Tennis ball exercise (Figure $5 \mathrm{~A}$ ); 4. Glass squeezing exercise (Figure $5 \mathrm{~B}$ ).

The exercises were performed at least three sessions a day. During the sessions, each exercise was performed with a sustained maximum contraction force held for 10 seconds and was repeated for six sets of 10 repetitions. For the patients with persistent pain in the TMC joint, concomitant use of an oral anti-inflammatory medication or intra-articular steroid injection alleviated the pain and allowed the exercises to continue without interruption.

With persistence in performing the two-pronged regimen, the key ligament, which is at the state of elastic deformation, will not be stretched any longer and, in time, its spontaneous shrinkage will take place while the stabilizing muscles become stronger. As a result, the base of the first metacarpal would return permanently to its anatomical position and the thumb and wrist would regain their normal postures (Figure 6).

Figure 3. Wrist and Base of the Thumb Position in Individuals Predisposed to Trapeziometacarpal Joint Laxity
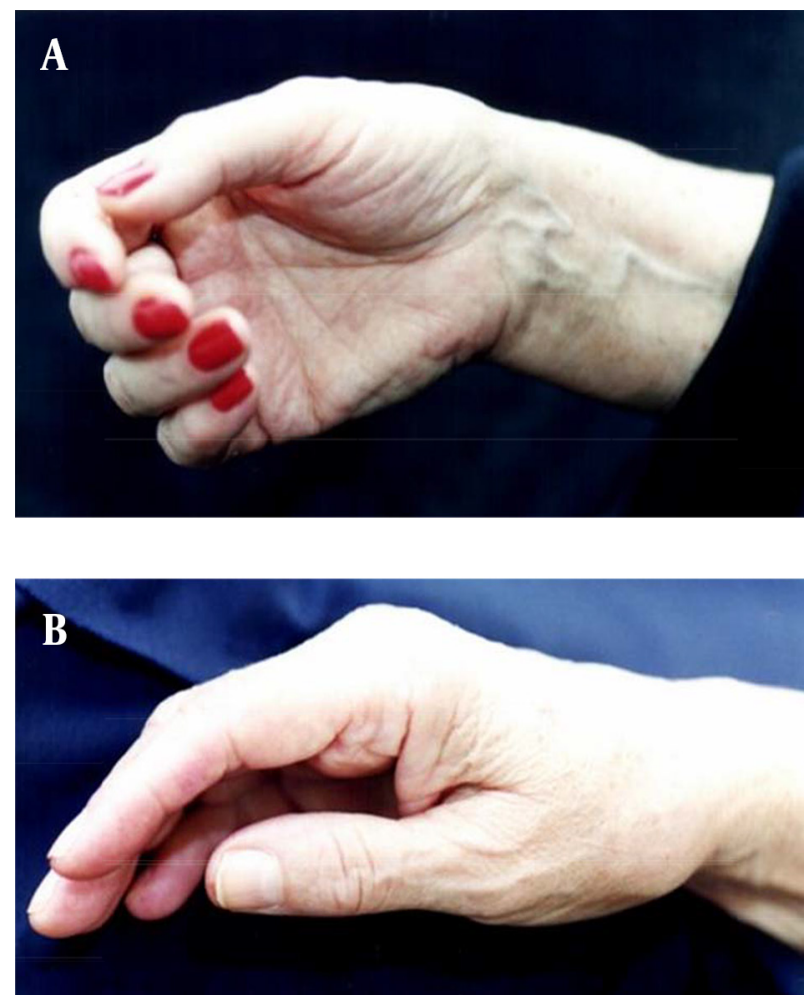

A, ulnar deviation of the wrist as a component of the habitual adducted posture of the thumb; B, "fork deformity" of the base of the thumb due to the subluxation of the first metacarpal at the TMC joint. 
Zahiri H et al.
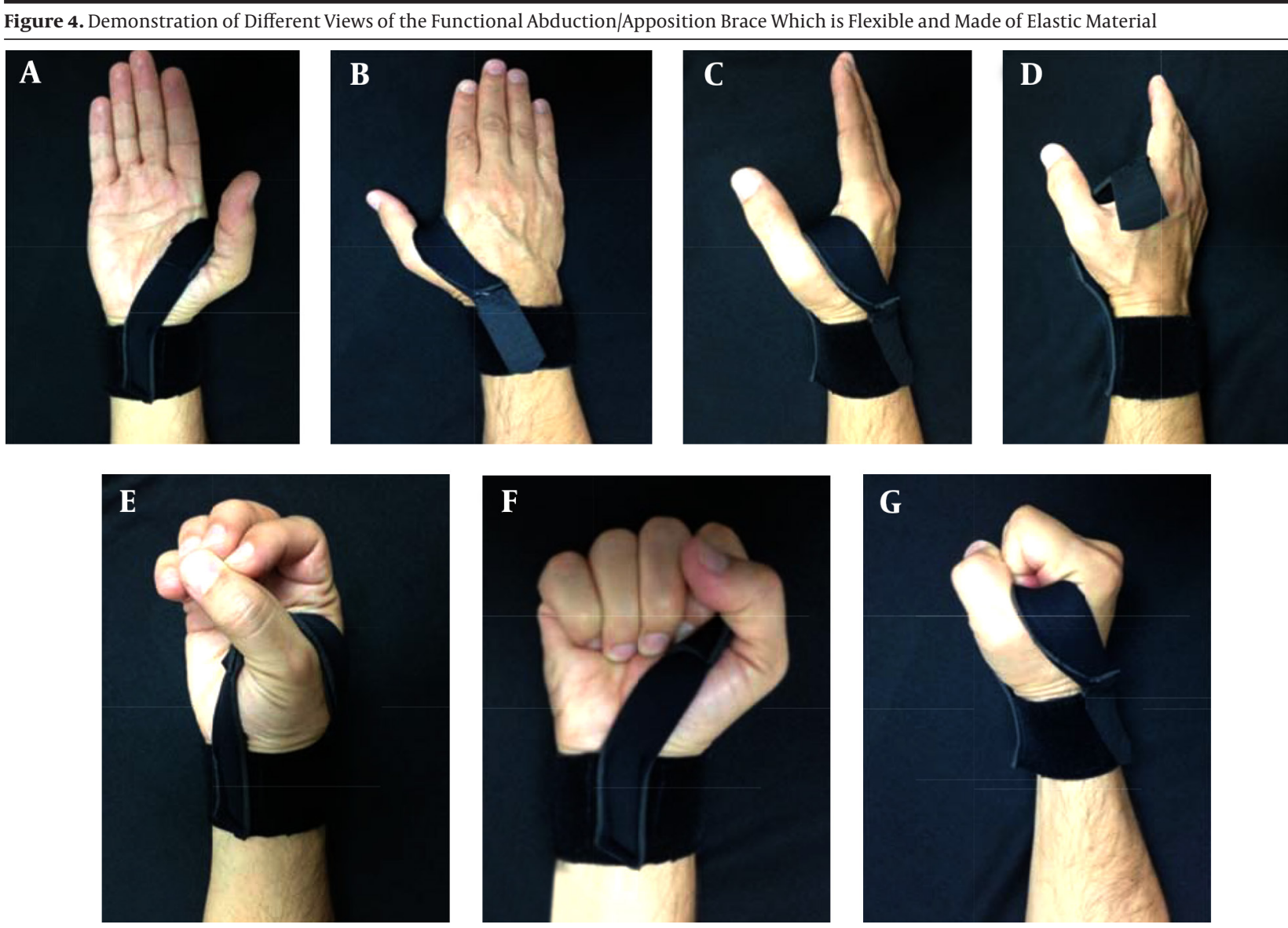

A, the volar view; B, the dorsal view; C, the side view; D, the brace has two components: one is a removable elastic wrist brace with Velcro attachment. The other is an elastic strap permanently attached to the volar aspect of the elastic wrist brace with its dorsal end left free for Velcro attachment to the dorsal aspect of the wrist elastic brace. This allows the thumb to freely move through a full range of opposition. For application, the strap is stretched to the patients' comfort while the thumb is securely maintained in abduction. E, demonstration of full apposition of the thumb against all fingers; F, volar view of full fist making; $\mathrm{G}$, lateral view of full fist making.

Figure 5. Strengthening of Dynamic Stabilizers of the Trapezio-Metacarpal Joint
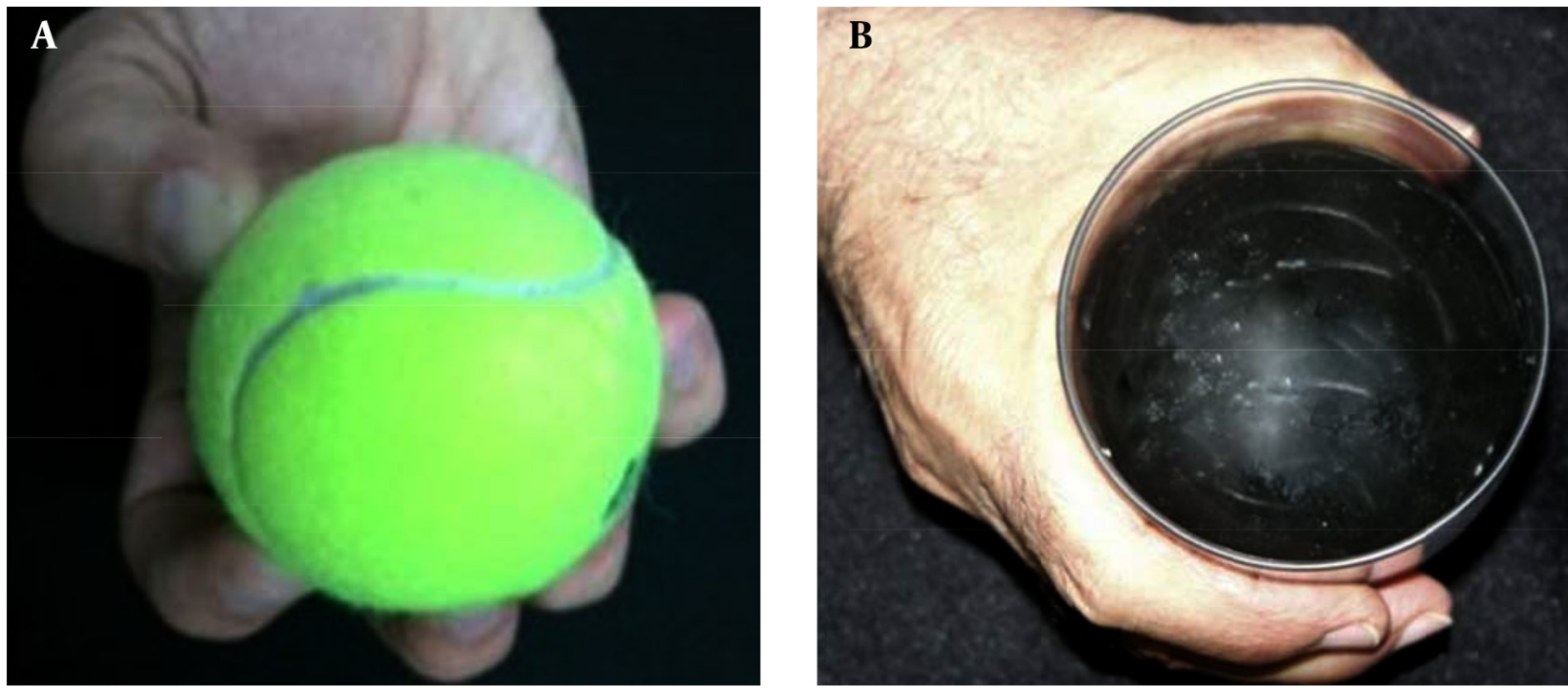

A, "tennis ball" exercise in which the pulps of four fingers and the thumb are spread around the waist of the ball with the thumb in full apposition/abduction; B, "glass squeezing" exercise in which the thumb is in full apposition/abduction while the glass remains in contact with the hand and fingers at all times. 
Figure 6. The Position of the Base of the First Metacarp Before and After Conservative Treatment

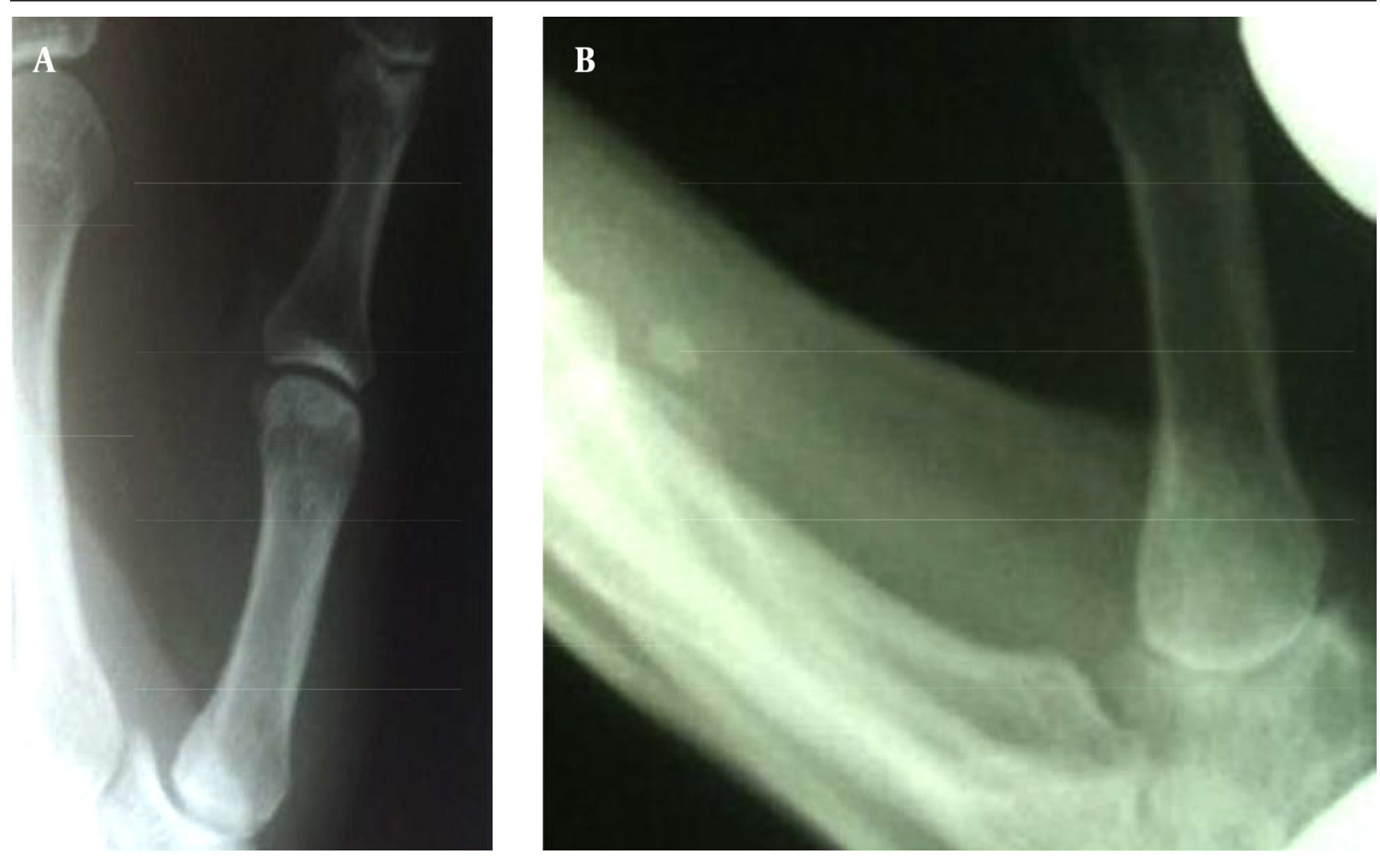

A, Roberts X-ray view of early stages of subluxation of the base of the TMC joint; B, the same patient with sustained reduction of the base of the first metacarpal after 47 months continued conservative treatment.

\section{Objectives}

Thus far the issue of causation for idiopathic osteoarthritis of the TMC joint has not been conclusively investigated and described in the literature. The objectives of this clinical investigation have been to delineate the cause of this saddle entity through its related anatomopathological and biomechanical considerations. Also, to present a new effective method for the prevention as well as its treatment at the early stages based on the findings.

\section{Materials and Methods}

All the patients gave informed consents prior to being included into the study. The study was authorized by the local ethical committee and was performed in accordance with the ethical standards of the 1964 Declaration of Helsinki as revised in 2000.

A retrospective study was performed on 17 patients diagnosed with the early stages of idiopathic TMC joint osteoarthritis in whom factors such as age, gender, occupation and physical habits involving the hands as well as the location and duration of the symptoms were considered. The patients were seen during a period beginning May 2000 and ending April 2010. The patients' evaluation included history of the symptoms, physical examination and radiographic studies as well as duration of the patients' follow-up. There was no history of a prior specific injury to either of the patients' thumbs. The patients' age ranged from 57 to 73 years with a mean of 66 years. There were 12 females and five males. Their initial symptom was pain in the base of the thumb, which increased with repeated or forceful use of the thumb. The duration of their symptom ranged 6 - 20 months. On examination, the TMC joint showed tenderness with a demonstrable laxity, allowing some degree of reproducible dorsoradial translation of the base of the first metacarpal. Roberts view X-rays taken revealed mild to slight joint subluxation with no clinically significant arthritic changes (stage I of Eaton-Littler classification or stage 0 of Comtet et al.) (19). The diagnosis was established through the history, symptoms, and clinical and radiographic objective findings.

The patients were treated with the use of a low profile functional abduction/apposition brace and specifically devised isometric exercises. The patients were checked monthly. A pain scoring system was used to quantify the level of their symptoms before their treatment was initiated and after the treatment was completed (Table 1).

The patients' follow-up period was started after they 
had become symptom free. Their mean follow-up period was 28.4 months.

\section{Results}

Before the treatment was initiated, the mean pain score for the patients was 3.6. After the completion of the treatment, all 17 patients were symptom free. The last Roberts X-ray view of all the patients' involved thumbs revealed anatomical reduction of the TMC joint (Figure 6). The patients' demographics are summarized as follows (Table 2):

Subsequent to the completion of the patients' conservative treatment, no patient showed recurrence of symptoms to require any treatment modality, including surgery.

\begin{tabular}{lr}
\hline Table 1. Pain Scoring System & \\
\hline Pain Score & Description \\
\hline $\mathbf{0}$ & No pain \\
$\mathbf{1}$ & Mild pain; no pain medication used \\
$\mathbf{2}$ & Slight to moderate intermittent pain; frequent use of nonprescription pain medication \\
$\mathbf{3}$ & Moderate intermittent pain with use of prescription pain medication \\
\hline $\mathbf{5}$ & Sever constant pain with frequent use of prescription pain medication \\
\hline
\end{tabular}

\begin{tabular}{|c|c|c|c|c|c|c|c|}
\hline Case Number & Gender & Age, $y$ & Job & $\begin{array}{l}\text { Symptoms } \\
\text { Period Before the } \\
\text { Treatment Initiated }\end{array}$ & $\begin{array}{l}\text { Pain Score } \\
\text { Before the } \\
\text { Treatment }\end{array}$ & $\begin{array}{l}\text { Pain Score } \\
\text { After the } \\
\text { Treatment }\end{array}$ & $\begin{array}{l}\text { Follow-up Period } \\
\text { After Patient Became } \\
\text { Symptom Free, mo }\end{array}$ \\
\hline 1 & Female & 70 & Housewife & 11 & 3 & 0 & 40 \\
\hline 2 & Female & 66 & $\begin{array}{l}\text { Telephone } \\
\text { operator }\end{array}$ & 6 & 3 & 0 & 47 \\
\hline 3 & Female & 69 & Housewife & 9 & 4 & 0 & 36 \\
\hline 4 & Male & 73 & Teacher & 20 & 3 & 0 & 34 \\
\hline 5 & Female & 64 & Housewife & 14 & 4 & 0 & 33 \\
\hline 6 & Female & 58 & $\begin{array}{c}\text { Dental } \\
\text { assistant }\end{array}$ & 6 & 3 & 0 & 42 \\
\hline 7 & Male & 57 & Teacher & 8 & 3 & 0 & 43 \\
\hline 8 & Female & 71 & Housewife & 12 & 4 & 0 & 32 \\
\hline 9 & Female & 66 & Hand writer & 18 & 4 & 0 & 47 \\
\hline 10 & Female & 64 & Dentist & 12 & 2 & 0 & 22 \\
\hline 11 & Male & 65 & Teacher & 10 & 3 & 0 & 14 \\
\hline 12 & Female & 69 & Psychiatrist & 14 & 4 & 0 & 12 \\
\hline 13 & Female & 69 & Housewife & 8 & 4 & 0 & 10 \\
\hline 14 & Male & 66 & Writer & 10 & 4 & 0 & 13 \\
\hline 15 & Female & 65 & Secretory & 12 & 4 & 0 & 17 \\
\hline 16 & Male & 66 & Teacher & 14 & 4 & 0 & 20 \\
\hline 17 & Female & 64 & Housewife & 12 & 5 & 0 & 20 \\
\hline
\end{tabular}

a Type of treatment is conservative treatment included thumb postural training through the use of a functional abduction/apposition brace, specifically devised isometric exercises and occasional use of steroid injection or oral nonsteroidal anti-inflammatory drugs (NSAIDs). 


\section{Discussion}

Spontaneous attenuation of the key stabilizing ligament of the TMC joint is currently accepted as the first pathological change which finally results in osteoarthritis of the joint. So far, no specific cause has been presented for this spontaneous attenuation of the anterior oblique (AO) or dorsoradial ligament, referred to as key stabilizing ligaments. A long-standing habitual adduction posture of the thumb is presented by the authors as the potential cause of the spontaneous attenuation of the key ligament. The long standing adducted posture of the thumb maintains the TMC joint key ligament at a relaxed position and allows its ongoing atrophy and attenuation. The position places the adductor pollicis muscle in mechanical advantage for a gradually occurring over activity of the muscle, the only destabilizing force of the TMC joint. Concomitantly, sustained adducted posture of the thumb causes progressive weakness of the TMC joint stabilizing muscles. As a result, the overly active adductor pollicis force would lever the base of the first metacarpal towards dorsoradial translation and subluxation. However, this theory is not yet proven and further studies are needed to substantiate the described theory.

So far, the focus of any conservative treatment for the early stages of the condition has been palliative, with no specific measure to stop or reverse the progressive pathology of the TMC joint. The authors emphasized on early detection of the habitual adduction posture of the thumb and presented effective conservative measures which can either eliminate the attenuation of the key ligament at its early stages or reverse the process of the joint laxity and subluxation before the attenuation reaches the state of plastic deformation.

To regain the normal posture of the thumb with sustained anatomical reduction of the TMC joint, a combination of specifically designed low profile functional brace and specifically devised isometric exercises were used. The authors postulated that the reason for reversal of the key ligament attenuation is the fact that at the early stages of the condition, the stretched and attenuated key ligament is at the state of reversible elastic deformation. Therefore, shrinkage of the ligament can occur through a sustained reduction of the TMC joint that maintains the key ligament at a relaxed position. The gradually contracted ligament, supported by the force of progressively strengthened stabilizing muscles, provides a permanently stable joint reduction. In the late stage of the condition, the stretched attenuated key ligament has already reached the irreversible state of plastic deformation. Therefore, only surgical reconstruction of the ligament, as reported by various authors can overcome the deficiency $(1,6,20-25)$.

In conclusion, encouraging results were obtained in reversal of the early stages of the TMC joint osteoarthritis in 17 symptomatic patients through the presented conser- vative method. Such results warrant the use of the method for the treatment of the early stages of the condition and for the prevention in all the individuals whose hands show the potential for developing the condition, as can be noted in their habitually adducted thumb posture.

\section{Authors' Contributions}

Hormoz Zahiri, Christopher Zahiri and Mansour Eghbali: study concept, design, drafting the manuscript. Christopher Zahiri and Mansour Eghbali: analysis and interpretation of data. Hormoz Zahiri: study supervision

\section{References}

1. Lane LB, Eaton RG. Ligament reconstruction for the painful "prearthritic" thumb carpometacarpal joint. Clin Orthop Relat Res. 1987(220):52-7.

2. Lin JD, Karl JW, Strauch RJ. Trapeziometacarpal joint stability: the evolving importance of the dorsal ligaments. Clin Orthop Relat Res. 2014;472(4):1138-45.

3. Pellegrini VJ. Pathomechanics of the thumb trapeziometacarpal joint. Hand Clin. 2001;17(2):175-84.

4. Imaeda T, An KN, Cooney W3, Linscheid R. Anatomy of trapeziometacarpal ligaments. J Hand Surg Am. 1993;18(2):226-31.

5. Pellegrini JVD. Osteoarthritis at the base of the thumb. Ortho Clin North Am. 1992;23(1):83-102.

6. Pelligrini VJ. Osteoarthritis of the trapeziometacarpal joint: the pathophysiology of articular cartilage degeneration. II. Articular wear patterns in the osteoarthritic joint. J Hand Surg Am. 1991;16(6):975-82.

7. Carroll RE. Arthrodesis of the carpometacarpal joint of the thumb. A review of patients with a long postoperative period. Clin Orthop Relat Res. 1987(220):106-10.

8. Dhar S, Gray IC, Jones WA, Beddow FH. Simple excision of the trapezium for osteoarthritis of the carpometacarpal joint of the thumb. J Hand Surg Br. 1994;19(4):485-8.

9. Molitor PJA, Emery RJH, Meggitt BF. First metacarpal osteotomy for carpo-metacarpal osteoarthritis. J Hand Surg. 1991;16(4):424-7.

10. Gomes Carreira AC, Jones A, Natour J. Assessment of the effectiveness of a functional splint for osteoarthritis of the trapeziometacarpal joint on the dominant hand: a randomized controlled study. J Rehabil Med. 2010;42(5):469-74.

11. Pellegrini VJ. Osteoarthritis of the trapeziometacarpal joint: the pathophysiology of articular cartilage degeneration. I. Anatomy and pathology of the aging joint. J Hand Surg Am. 1991;16(6):967-74.

12. Pellegrini VJ, Olcott CW, Hollenberg G. Contact patterns in the trapeziometacarpal joint: the role of the palmar beak ligament.J Hand Surg Am. 1993;18(2):238-44.

13. Ferrari B, Steffee AD. Trapeziometacarpal total joint replacement using the Steffee prosthesis. J Bone Joint Surg Am. 1986; 68(8):1177-84.

14. Kauer JMG. Functional Anatomy of the Carpometacarpal: Joint of the Thumb. Clin Orthop Relat Res. 1987;220:7-13.

15. Glickel SZ. Clinical assessment of the thumb trapeziometacarpal joint. Hand Clin. 2001;17(2):185-95.

16. Pomerance JF. Painful basal joint arthritis of the thumb. Part I: Anatomy, pathophysiology, and diagnosis. Am J Ortho Surg. 1995;24(5):401-8.

17. Burton RI, Pellegrini VD. Basal joint arthritis of thumb. J Hand Surg. 1987;12(4):331-48.

18. Eaton RG, Glickel SZ. Trapeziometacarpal osteoarthritis. Staging as a rationale for treatment. Hand Clin. 1987;3(4):455-71.

19. Comtet JJ, Gazarian A, Fockens W. [Definition and classification of basal joint osteoarthritis. A critical analysis and proposals. Treatment options]. Chir Main. 2001;20(1):5-10.

20. Eaton RG, Littler JW. Ligament reconstruction for the painful thumb carpometacarpal joint. J Bone Joint Surg Am. 1973;55(8):1655-66. 


\section{Zahiri Het al.}

21. Eaton RG, Lane LB, Littler JW, Keyser JJ. Ligament reconstruction for the painful thumb carpometacarpal joint: a long-term assessment. J Hand Surg Am. 1984;9(5):692-9.

22. Froimson AI. Tendon interposition arthroplasty of carpometacarpal joint of the thumb. Hand Clin. 1987;3(4):489-505.

23. Pomerance JF. Painful basal joint arthritis of the thumb. Part II: Treatment. Am J Orthop (Belle Mead NJ). 1995;24(6):466-72.
24. Menon J. The problem of trapeziometacarpal degenerative arthritis. Clin Orthop Relat Res. 1983(175):155-65.

25. Raven EE, Kerkhoffs GM, Rutten S, Marsman AJ, Marti RK, Albers GH. Long term results of surgical intervention for osteoarthritis of the trapeziometacarpal joint : comparison of resection arthroplasty, trapeziectomy with tendon interposition and trapezio-metacarpal arthrodesis. Int Orthop. 2007;31(4):547-54. 